\title{
SAMHD1 knockout mice: modeling retrovirus restriction in vivo
}

\author{
$\mathrm{Li} \mathrm{Wu} u^{1,2,3}$
}

\begin{abstract}
The host dNTP hydrolase SAMHD1 acts as a viral restriction factor to inhibit the replication of several retroviruses and DNA viruses in non-cycling human immune cells. However, understanding the physiological role of mammalian SAMHD1 has been elusive due to the lack of an animal model. Two recent studies reported the generation of samhd1 knockout mouse models for investigating the restriction of HIV-1 vectors and endogenous retroviruses in vivo. Both studies suggest that SAMHD1 is important for regulating the intracellular dNTP pool and the intrinsic immunity against retroviral infection, despite different outcomes of HIV-1 vector transduction in these mouse models. Here I discuss the significance of these new findings and the future directions in studying SAMHD1-mediated retroviral restriction.
\end{abstract}

\section{Introduction}

The dNTP hydrolase SAMHD1 functions as a retroviral restriction factor in human non-cycling myeloid cells and resting $\mathrm{CD}^{+}{ }^{+} \mathrm{T}$-cells [1-7]. The molecular mechanisms by which SAMHD1 restricts retrovirus replication in non-cycling cells are not fully understood. Although SAMHD1-mediated HIV-1 restriction involves the depletion of the intracellular dNTP pool in non-cycling cells $[4,5,7]$, recent studies revealed that phosphorylation of human SAMHD1 protein negatively regulates its HIV-1 restriction function [8-10]. The lack of SAMHD1 protein expression in humans due to $S A M H D 1$ gene mutations can result in a rare, but severe autoimmune disease called Aicardi-Goutieres syndrome (AGS). AGS is associated with elevated type I interferon (IFN) production and likely is due to an innate immune response to accumulated nucleic acids in the cell [11]. Despite the rapid research progress in understanding the molecular mechanisms of SAMHD1-mediated retroviral restriction, characterization of the physiological role of SAMHD1 in vivo has been elusive due to the lack of an animal model.

Generating a samhd1 knockout (KO) mouse model can help elucidate the physiological functions of

\footnotetext{
Correspondence: wu.840@osu.edu

${ }^{1}$ Center for Retrovirus Research, Department of Veterinary Bioscience, 1900 Coffey Road, Columbus, OH 43210, USA

2Department of Microbial Infection and Immunity, Columbus, OH 43210, USA Full list of author information is available at the end of the article
}

SAMHD1 in vivo [12,13]. Alternative splicing generates two isoforms of mouse SAMHD1 (mSAMHD1) proteins [12], which differ at the C-terminus and share about 72$74 \%$ identity with human SAMHD1 (hSAMHD1). mSAMHD1 possesses a similar dNTP hydrolase activity and also restricts in vitro HIV-1 infection as hSAMHD1 [4]. Both mSAMHD1 isoforms can block in vitro HIV-1 or SIV infections when they are ectopically expressed in a human monocytic cell line (U937) that does not express endogenous hSAMHD1 and is differentiated into non-cycling, macrophage-like cells [4]. Interestingly, two recent studies reported the generation of samhd1 $\mathrm{KO}$ mouse models for studying retroviral restriction in vivo, while the effects of SAMHD1 deficiency on HIV-1 vector transduction in these mice appeared to be distinct $[12,13]$. The new findings and implications of these two studies are analyzed and discussed below.

\section{New findings and implications \\ Generation of samhd1-null mice}

Two studies by Rehwinkel et al. and Behrendt et al. generated conditional knockout strains in a mixed or a pure C57BL/6 background and obtained the samhd1-null mice by deleting exon 2 of the gene [12,13]. In both cases, homozygous samhd1-null mice had no detectable amount of SAMHD1 protein in bone marrow-derived dendritic cells (BM-DCs), primary mouse embryonic fibroblasts (MEFs), or splenocytes [12,13]. It is possible that these two samhd1-null mouse strains may have 
minor differences due to different approaches used in these studies $[12,13]$. Unlike the severe autoimmune disease in SAMHD1-deficient human patients with AGS [11], the samhd1-null mice were healthy beyond the age of 96 weeks [12] or up to 70 weeks [13], respectively. Neither SAMHD1-deficient mouse strain developed detectable pathology or autoimmunity [12,13], suggesting that mSAMHD1 function might be redundant or not directly involved in the innate immune responses to nucleic acids in mice. It also is conceivable that other triggers (such as cytokines, dysfunction of immune cells, or environmental factors) in human AGS patients may contribute to the disease pathogenesis in addition to the genetic deficiency of SAMHD1.

\section{Increased intracellular dNTP pool in samhd1-null mice}

Using the samhd1-null mouse models, both studies confirmed that mSAMHD1 acts as a dNTP hydrolase in vivo to reduce intracellular dNTP concentrations in multiple cell types [12,13] (Figure 1). Compared with cells from wild-type mice, the intracellular dNTP concentrations were significantly increased in BM-DCs, bone marrow-derived macrophages (BM-DMs) [12,13], B-cells, T-cells, and MEFs [13] derived from samhd1 $1^{-/-}$ mice. For example, the intracellular dTTP levels in BMDCs from samhd1 $1^{-/-}$mice were quantified in both studies using different methods and showed an approximately 5- fold increase compared with those from wild-type mice $[12,13]$. Of note, the dTTP level in BM-DCs $(0.5 \mu \mathrm{M})$ from wild-type mice reported by Rehwinkel et al. was 5 -fold higher than that in human monocyte-derive DCs $(\sim 0.1 \mu \mathrm{M})$ from healthy donors [7]. This comparison suggests that dNTP levels in mouse cells are likely higher than those in human cells; however, it is difficult to directly compare these results since different approaches and cell types were used in independent experiments.

\section{Induction of type I IFN-stimulated genes in samhd1-null mice}

Spontaneous and significant induction of mRNA expression of IFN-stimulated genes (ISGs) was observed in multiple cell types and tissues from samhd1-null mice compared with wild-type mice $[12,13]$. These results and the previous study by Rice et al. [11] suggest that SAMHD1 is a negative regulator in suppressing IFNinduced innate immune responses (Figure 1). Using a sensitive IFN bioassay, Rehwinkel et al. showed increased mRNA expression of several ISGs and the proinflammatory cytokine TNF $\alpha$ in spleens, macrophages and fibroblasts from samhd1-null mice compared with those from wild-type mice [12]. However, SAMHD1deficient mice did not display detectable amounts of circulating IFN proteins or upregulation of ISG products in their sera or most tissues examined [12]. Using

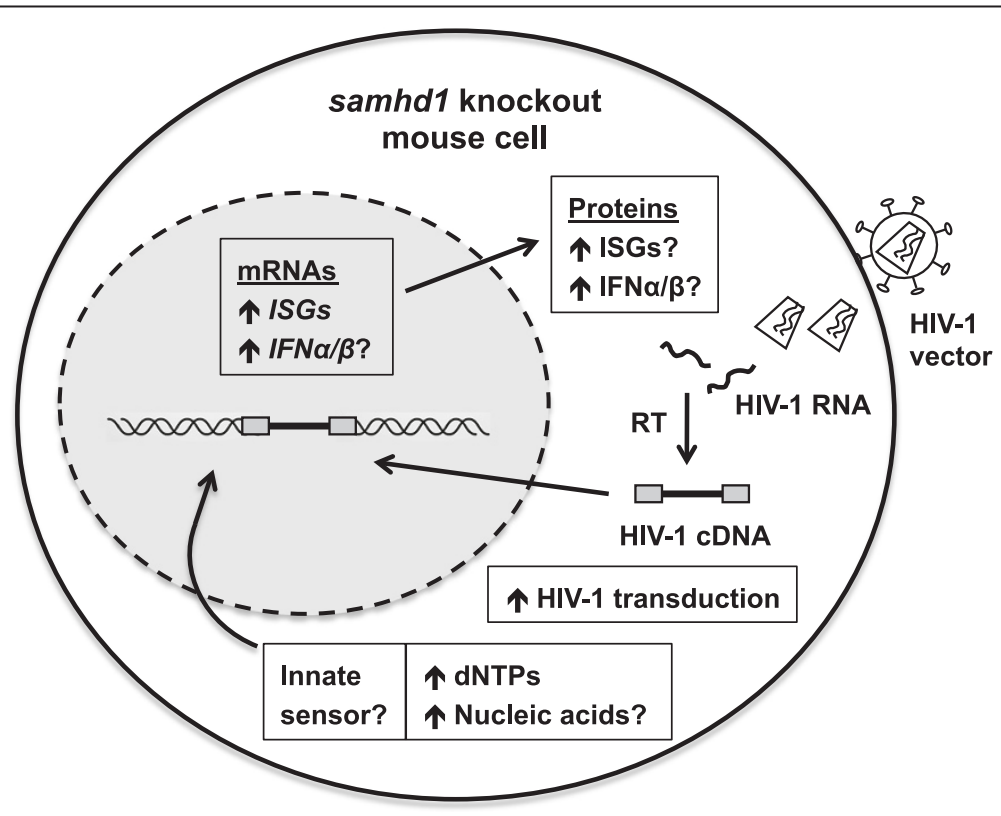

Figure 1 HIV-1 infection and intrinsic immune response in SAMHD1-deficient mouse cells. Intracellular dNTP concentrations are significantly increased in multiple cell types from samhd1-null mice compared with control cells, indicating that mSAMHD1 acts as a dNTP hydrolase in vivo to reduce the intracellular dNTP pool. Spontaneous transcriptional induction of type I IFN-stimulated genes (ISGs) has been observed in several cell types and tissues from samhd1-null mice compared with wild-type mice. Therefore, mSAMHD1 might play a role in regulating the IFN signaling pathway in vivo, although the innate sensor that triggers the spontaneous ISG responses is unclear. Transduction of SAMHD1-deficient mice or derived cells with HIV-1 vectors is increased relative to wild-type mice or murine cells, which appears to be dependent on the affinity for dNTPs of the HIV-1 RT and the experimental conditions in these studies (refer to Table 1). RT, HIV-1 reverse transcriptase. 
transcriptome sequencing, Behrendt et al. demonstrated that 123 ISGs were significantly up-regulated ( $85 \%$ of 141 genes showed signficiant up-regulation) in SAMHD1deficient BM-DMs compared with those from control mice [13]. The up-regulation of ISG transcripts was dependent on IFN $\beta$ since crossing SAMHD1- and IFN $\beta$ deficient mice prevented spontanous transcriptional activation of the ISGs [13]. Compared with wild-type mouse MEFs or BM-DMs, no difference of INF $\alpha$ induction was observed in SAMHD1-deficient cells treated with in vitrotranscribed RNA [12]. Notably, the undetectable type I IFN protein in the sera from SAMHD1-deficient mice [12] did not mirror the elevated levels of serum IFN $\alpha$ found in some human AGS patients [3,11], suggesting that SAMHD1 deficiency in mice is not sufficient to enhance the production or release of type I IFNs. Furthermore, similar levels of the induced INF $\alpha$ were detected in the sera of wild-type and SAMHD1-deficient mice infected with encephalomyocarditis virus, an RNA virus that can trigger INF $\alpha$ production [12]. These data suggest that mSAMHD1 deficiency is not sufficient to trigger IFN in response to nucleic acids or RNA virus infection in mice.

Increased HIV-1 vector transduction in samhd1-null mice Human blood monocytes and resting $\mathrm{CD} 4^{+} \mathrm{T}$-cells from SAMHD1-deficient AGS patients are more susceptible to in vitro HIV-1 infection compared with normal cells $[3,5,6]$, suggesting that hSAMHD1 contributes to HIV-1 restriction in non-cycling immune cells through an intrinsic mechanism. Despite significantly increased intracellular dNTP levels in SAMHD1-deficient mouse cells,
Rehwinkel et al. did not observe any changes in singlecycle infection or transduction with HIV-1 or Moloney murine leukemia virus (Mo-MLV) vectors in samhd1-null mice or derived cells when compared with wild-type mice or cells [12]. Rehwinkel et al. interpreted that the lack of HIV-1 restriction in wild-type mouse cells was likely attributable to higher dNTP concentrations $(\sim 0.5 \mu \mathrm{M})$ than the $\mathrm{K}_{\mathrm{M}}(\sim 0.07 \mu \mathrm{M})$ of HIV-1 reverse transcriptase (RT). However, a $\sim 5$-fold increase in HIV-1 in vitro infection was evident in BM-DMs, BM-DCs and MEFs from samhd1-null mice using a vector expressing a mutant HIV-1 RT (V148I), which required higher levels of dNTPs relative to wild-type RT [12]. In line with this observation, Lahouassa et al. showed that the V148I RT mutant HIV-1 was approximately 6-fold less infectious in SAMHD1sufficient human monocyte-derived macrophages compared with wild-type HIV-1 [4]. Furthermore, compared with wild-type mice, in vivo infection of samhd1-null mice with the mutant HIV-1 vector increased the viral transduction of splenocytes by $\sim 8$-fold and significantly enhanced transduction of myeloid and lymphoid cells as well as non-hematopoietic cells [12]. These results suggest that mSAMHD1 inhibits in vivo HIV-1 vector transduction by reducing the intracellular dNTP pool, which is in agreement with previous in vitro studies indicating that overexpressed mSAMHD1 blocks HIV-1 infection in differentiated human monocytic U937 cells [4].

In contrast to no effect on the transduction of samhd1-null mice with an HIV-1 vector expressing a wild-type RT reported by Rehwinkel et al. [12], Behrendt et al. used a different HIV-1 vector expressing a wildtype RT (Table 1) and demontrated a $\sim 3$ - to 8-fold

Table 1 Comparison of viral infections of samhd1 $\mathrm{KO}$ and wild-type mice and derived cells $[12,13]$

\begin{tabular}{|c|c|c|c|}
\hline & & Rehwinkel et al. [12] & Behrendt et al. [13] \\
\hline \multicolumn{2}{|c|}{$\begin{array}{l}\text { In vitro HIV-1 vector infection of cells from samhd1 } \mathrm{KO} \text { mice compared } \\
\text { with wild-type mice }\end{array}$} & $\begin{array}{l}\text { - No effect on the infectivity of HIV-1 } \\
\text { vector (wild-type RT) at 1-2 dpi* } \\
\text { - 5-fold increase of HIV-1 RT mutant } \\
\text { (V148I) vector infection in BM-DC, } \\
\text { BM-DMs, and MEFs }\end{array}$ & $\begin{array}{l}\text { - } 5 \text {-fold increased infectivity } \\
\text { of HIV-1 vector (wild-type RT) } \\
\text { in BM-DCs at } 3 \mathrm{dpi}^{*} \\
\text { - Likely dependent on HIV-1 } \\
\text { vector strains }\end{array}$ \\
\hline \multirow[t]{3}{*}{$\begin{array}{l}\text { In vivo HIV-1 vector transduction of samhd } 1 \mathrm{KO} \text { mice } \\
\text { compared with wild-type mice }\end{array}$} & $\begin{array}{l}\text { Effects on HIV-1 } \\
\text { vector transduction }\end{array}$ & $\begin{array}{l}\text { - No effect on HIV-1 vector (wild-type RT) } \\
\text { at } 5-6 \mathrm{dpi}^{*} \\
\text { - 3-8-fold increase of HIV-1 RT mutant } \\
\text { (V148I) in multiple cell types at 5-6 dpi* }\end{array}$ & $\begin{array}{l}\text { - 4-fold increased HIV-1 vector } \\
\text { transduction (wild-type RT) } \\
\text { in multiple cell types at } 3 \mathrm{dpi}^{*}\end{array}$ \\
\hline & $\begin{array}{l}\text { HIV-1 viral vector } \\
\text { used }\end{array}$ & $\begin{array}{l}\text { - pRRLsin.eGFP/ pCMVD8.2 vector } \\
\text { (encoding Vif, Vpr, Vpu, and Nef) } \\
\text { • HIV-1-based pCSGW/ p8.91 vector } \\
\text { (no accessory genes) }\end{array}$ & $\begin{array}{l}\text { - HIV-1 } \text { NL4-3 }_{\text {based GFP reporter }} \\
\text { vector (pHR.CMVGFP/ } \\
\text { pCMVDR8.91) }\end{array}$ \\
\hline & $\begin{array}{l}\text { Amount of HIV-1 } \\
\text { vector injected }\end{array}$ & $\begin{array}{l}\text { - Intravenously with } 5 \times 10^{7} \text { or } 1 \times 10^{8} \\
293 T \text { cell infectious units }\end{array}$ & $\begin{array}{l}\text { - Intravenously with } 5 \times 10^{6} \\
\text { viral particles }\end{array}$ \\
\hline \multicolumn{2}{|l|}{ Exogenous Mo-MLV replication } & No effect & ND\# \\
\hline \multicolumn{2}{|l|}{ Friend virus infection (a type of MLV) } & $N D^{\#}$ & No effect \\
\hline \multicolumn{2}{|c|}{ Endogenous Mo-MLV replication or mouse retrotransposons } & No effect & ND \\
\hline \multicolumn{2}{|c|}{ Encephalomyocarditis virus infection (a mouse RNA virus that induces IFNa) } & No effect & ND \\
\hline
\end{tabular}

*dpi, days post-infection; ${ }^{\#} \mathrm{ND}$ : not done. 
increase of in vivo transduction of splenocytes, $\mathrm{CD} 4^{+}$ and $\mathrm{CD}^{+} \mathrm{T}$-cells, B-cells, dendritic cells, and macrophages in samhd1-null mice compared with those in wild-type mice [13]. Moreover, in vitro infection of BMDCs from samhd1-null mice using two different HIV-1 vectors expressing wild-type $\mathrm{RT}$ also showed a $\sim 5$-fold increase compared with control cells. Reconstitution of mSAMHD1 expression in BM-DCs from samhd1-null mice restored HIV-1 restriction, suggesting that mSAMHD1 contributes to the intrinsic anti-HIV-1 mechanisms in vivo, at least in part [13]. The discrepancy in results of HIV-1 transduction in these two strains of SAMHD1-deficient mice might be attributed to the different experimental approaches and conditions used in these studies [12,13] (Table 1). The viral infections and transductions in SAMHD1-deficient mice or derived cells reported in these two studies are compared in Table 1.

\section{SAMHD1 does not affect endogenous retroviruses or retrotransposons in mice}

It is conceivable that SAMHD1 may block endogenous retroviruses or retrotransposons by reducing the intracellular dNTP pool through its hydrolase activity. Rehwinkel et al. showed that SAMHD1 deficiency did not affect RNA levels of mouse long terminal repeat (LTR) retrotransposons and endogenous retroviruses (including MusD, IAP, Mu-ERV-L and m-poly-MLV) or the replication of endogenous Mo-MLV in BM-DCs and MEFs, or in lung and spleen tissues [12]. Therefore, mSAMHD1 appears to be independent of in vivo control of endogenous retroviruses and LTR retrotransposons in mice [12]. However, it is also possible that SAMHD1 may inhibit other retrotransposons or endogenous retroviruses in humans or mice. A recent study by Zhao et al. reported that diverse mammalian SAMHD1 proteins potently inhibited the non-LTR retrotransposon LINE-1 and LINE-1-mediated Alu/SVA retrotransposition in dividing human cell lines, suggesting that SAMHD1 may act as a cellular regulator of the conserved LINE-1 activity in mammals [14].

\section{Conclusions}

The studies by Rehwinkel et al. and Behrendt et al. have established samhd1-null mouse models to better understand the physiological role of SAMHD1 in vivo $[12,13]$. Although SAMHD1 is not essential for mouse survival or development, it functions as a dNTP hydrolase in vivo and reduces the intracellular dNTP pool. The new findings from these studies suggest that mSAMHD1 can restrict lentiviruses in vivo through an evolutionarily conserved intrinsic mechanism, by decreasing intracellular dNTP concentrations [12,13]. Moreover, SAMHD1 deficiency in mice results in IFN $\beta$-dependent transcriptional up-regulation of type I IFN-inducible genes and type I IFN signature in certain tissues and cell types [12,13], suggesting an important role of SAMHD1 in regulating innate immunity. The SAMHD1-deficient mouse model could be used as a potential tool to investigate the mechanisms of pathogenic type I IFN responses in autoimmune diseases in humans $[12,13]$.

\section{Future directions}

The molecular mechanisms underlying mSAMHD1mediated restriction of HIV-1 in vivo are only starting to be investigated. Previous in vitro studies using cultured human myeloid cells and resting $\mathrm{CD}^{+}{ }^{+} \mathrm{T}$-cells have demonstrated that hSAMHD1 inhibits the accumulation of late reverse transcription products of HIV-1 [1,2,4-7], which is correlated with the reduced intracellular dNTP pool in these non-cycling cells $[4,5,7]$. It is important to know whether the increased HIV-1 gene expression in cells derived from samhd1-null mice is due to the increased levels of late reverse transcription products during HIV-1 cDNA synthesis, which has not been addressed in either study using SAMHD1-deficient mice [12,13]. However, current evidence suggests that decreasing the intracellular dNTP levels by SAMHD1 might be necessary, but not sufficient for HIV-1 restriction [8-10]. Recent studies indicate that cell differentiation-dependent phosphorylation of hSAMHD1 at residue T592 negatively regulates its anti-HIV-1 function [8-10], but not dNTPase activity $[9,10]$. It would be interesting to know whether phosphorylation of mSAMHD also affects its hydrolase activity and anti-retroviral function.

The cellular sources of type I IFN and the innate sensors that trigger the spontaneous IFN response in SAMHD1-deficient mice remain unclear [12,13]. Comparing cells types that produce type I IFNs in the various KO mouse models of AGS-associated genes and cells from human AGS patients can provide a means to identify the cellular sources of IFN production and the molecular mechanisms underlying spontaneous IFN induction in SAMHD1-deficient mice. These studies may help reveal the cellular and molecular mechanisms by which SAMHD1 modulates IFN and ISG expression.

SAMHD1-mediated HIV-1 restriction in human myeloid cells and resting CD4 ${ }^{+} \mathrm{T}$-cells has been proposed as an immune evasion strategy for HIV-1 to avoid antiviral innate and adaptive immune responses and establish persistent infection [1,5,7]. SAMHD1 and Vpx interactions may contribute to the distinct pathogenic outcomes of HIV-1 and HIV-2 infection in humans. Additional in vivo studies using the macaque model and SIV infection as well as the humanized mouse model and HIV-1 infection could be useful to test this hypothesis.

Interestingly, recent genome-wide sequencing projects identified low frequencies of samhd1 somatic mutations 
in several types of human cancer, including leukemia, lung adenocarcinoma, myeloma, medulloblastoma, glioblastoma, pancreatic, and breast and colorectal cancers (discussed in [12,15]). de Silva et al. reported significant down-regulation of SAMHD1 expression in peripheral blood mononuclear cells from T-cell lymphoma patients compared with those from health donors, which correlated with significantly increased levels of samhd 1 promoter methylation [15]. These studies suggest a potential role of SAMHD1 silencing in cancer development and the involvement of epigenetic mechanisms in regulating SAMHD1 expression. Therefore, in conjunction with other gene $\mathrm{KO}$ mouse models targeting tumor suppressors, the SAMHD1-deficient mouse models provide an important tool to explore the potential role of SAMHD1 in cancer.

\begin{abstract}
Abbreviations
SAMHD1: Sterile alpha motif domain- and HD domain-containing protein 1; hSAMHD1: Human SAMHD1; mSAMHD1: Mouse SAMHD1; AGS: AicardiGoutieres syndrome; KO: Knockout; BM-DCs: Bone marrow-derived dendritic cells; BM-DMs: Bone marrow-derived macrophages; MEFs: Mouse embryonic fibroblasts; HIV-1: Human immunodeficiency virus type 1; HIV-2: Human immunodeficiency virus type 2; Mo-MLV: Moloney murine leukemia virus; SIV: Simian immunodeficiency virus; dNTP: Deoxynucleoside triphosphate; dNTPase: dNTP triphosphohydrolase; IFN: Interferon; ISG: IFN-stimulated gene; LINE-1: Long interspersed element-1.
\end{abstract}

\section{Competing interests}

The author declares that he has no competing interests.

\section{Acknowledgements}

The author thanks members of his laboratory for stimulating discussions and Dr. Vincenzo Coppola for helpful discussions about the techniques used in generating samhd 1 knockout mice in the published studies. The author thanks Dr. Kathleen Hayes-Ozello for critical reading and editing of the manuscript. The research in the author's laboratory is supported by grants (Al104483, Al098524 and Al102822) from the NIH and by the Public Health Preparedness for Infectious Diseases program of The Ohio State University. The author apologizes to many whose interesting work could not be cited due to space constraints (Viewpoints are limited to 15 references).

\section{Author details}

${ }^{1}$ Center for Retrovirus Research, Department of Veterinary Bioscience, 1900 Coffey Road, Columbus, OH 43210, USA. ${ }^{2}$ Department of Microbial Infection and Immunity, Columbus, OH 43210, USA. ${ }^{3}$ Comprehensive Cancer Center, The Ohio State University, Columbus, OH 43210, USA.

Received: 16 September 2013 Accepted: 14 November 2013

Published: 20 November 2013

\section{References}

1. Laguette N, Sobhian B, Casartelli N, Ringeard M, Chable-Bessia C, Segeral E, Yatim A, Emiliani S, Schwartz O, Benkirane M: SAMHD1 is the dendriticand myeloid-cell-specific HIV-1 restriction factor counteracted by Vpx. Nature 2011, 474(7353):654-657.

2. Hrecka K, Hao C, Gierszewska M, Swanson SK, Kesik-Brodacka M, Srivastava S, Florens L, Washburn MP, Skowronski J: Vpx relieves inhibition of HIV-1 infection of macrophages mediated by the SAMHD1 protein. Nature 2011, 474(7353):658-661

3. Berger A, Sommer AF, Zwarg J, Hamdorf M, Welzel K, Esly N, Panitz S, Reuter A, Ramos I, Jatiani A, et al: SAMHD1-deficient CD14+ cells from individuals with Aicardi-Goutieres syndrome are highly susceptible to HIV-1 infection. PLoS Pathog 2011, 7(12):e1002425.

4. Lahouassa H, Daddacha W, Hofmann H, Ayinde D, Logue EC, Dragin L, Bloch N, Maudet C, Bertrand M, Gramberg T, et al: SAMHD1 restricts the replication of human immunodeficiency virus type 1 by depleting the intracellular pool of deoxynucleoside triphosphates. Nat Immunol 2012, 13(3):223-228.

5. Baldauf HM, Pan X, Erikson E, Schmidt S, Daddacha W, Burggraf $M$ Schenkova K, Ambiel I, Wabnitz G, Gramberg T, et al: SAMHD1 restricts HIV-1 infection in resting CD4(+) T cells. Nat Med 2012, 18(11):1682-1687.

6. Descours B, Cribier A, Chable-Bessia C, Ayinde D, Rice G, Crow Y, Yatim A, Schwartz O, Laguette N, Benkirane M: SAMHD1 restricts HIV-1 reverse transcription in quiescent CD4(+) T-cells. Retrovirology 2012, 9:87.

7. St Gelais C, de Silva S, Amie SM, Coleman CM, Hoy H, Hollenbaugh JA, Kim B, Wu L: SAMHD1 restricts HIV-1 infection in dendritic cells (DCs) by dNTP depletion, but its expression in DCs and primary CD4+ T-lymphocytes cannot be upregulated by interferons. Retrovirology 2012, 9:105.

8. Cribier A, Descours B, Valadao AL, Laguette N, Benkirane M: Phosphorylation of SAMHD1 by cyclin A2/CDK1 regulates its restriction activity toward HIV-1. Cell Rep 2013, 3(4):1036-1043.

9. White TE, Brandariz-Nunez A, Valle-Casuso JC, Amie S, Nguyen LA, Kim B, Tuzova M, Diaz-Griffero F: The retroviral restriction ability of SAMHD1, but not its deoxynucleotide triphosphohydrolase activity, is regulated by phosphorylation. Cell Host Microbe 2013, 13(4):441-451.

10. Welbourn S, Dutta SM, Semmes OJ, Strebel K: Restriction of Virus Infection but Not Catalytic dNTPase Activity Is Regulated by Phosphorylation of SAMHD1. J Virol 2013, 87(21):11516-11524.

11. Rice GI, Bond J, Asipu A, Brunette RL, Manfield IW, Carr IM, Fuller JC, Jackson RM, Lamb T, Briggs TA, et al: Mutations involved in Aicardi-Goutieres syndrome implicate SAMHD1 as regulator of the innate immune response. Nat Genet 2009, 41(7):829-832.

12. Rehwinkel J, Maelfait J, Bridgeman A, Rigby R, Hayward B, Liberatore RA, Bieniasz PD, Towers GJ, Moita LF, Crow YJ, et al: SAMHD1-dependent retroviral control and escape in mice. EMBO J 2013, 32(18):2454-2462.

13. Behrendt R, Schumann T, Gerbaulet A, Nguyen LA, Schubert N, Alexopoulou D, Berka U, Lienenklaus S, Peschke K, Gibbert K, et al: Mouse SAMHD1 has antiretroviral activity and suppresses a spontaneous cell-intrinsic antiviral response. Cell Rep 2013, 4(4):689-696.

14. Zhao K, Du J, Han X, Goodier JL, Li P, Zhou X, Wei W, Evans SL, Li L, Zhang W, et al: Modulation of LINE-1 and Alu/SVA Retrotransposition by AicardiGoutieres Syndrome-Related SAMHD1. Cell Rep 2013, 4(6):1108-1115.

15. de Silva S, Wang F, Hake TS, Porcu P, Wong HK, Wu L: Downregulation of SAMHD1 expression correlates with promoter DNA methylation in Sezary syndrome patients. J Invest Dermatol 2013. doi: 10.1038/ jid.2013.1311. [Epub ahead of print on July 24, 2013]

doi:10.1186/1742-4690-10-142

Cite this article as: Wu: SAMHD1 knockout mice: modeling retrovirus restriction in vivo. Retrovirology 2013 10:142.

\section{Submit your next manuscript to BioMed Central and take full advantage of:}

- Convenient online submission

- Thorough peer review

- No space constraints or color figure charges

- Immediate publication on acceptance

- Inclusion in PubMed, CAS, Scopus and Google Scholar

- Research which is freely available for redistribution 